\title{
Clinical knowledge in OAB: introduction
}

\author{
Jacques Corcos, MD, FRCSC
}

Professor of Surgery/Urology, McGill University, Director of the Urology Unit, Shriners' Hospital, Director of Urology Unit, Rehabilitation Institute of Montréal, Montréal, QC

Cite as: Can Urol Assoc J 2011;5(5Supp|2):S134; D01:10.5489/cuaj.11197

A discussion of the clinical aspects of overactive bladder $(\mathrm{OAB})$ comprises this section of outstanding review articles.

The definition of the condition, $\mathrm{OAB}$, has evolved over time. Urgency in particular, the most bothersome symptom, is relatively easy to define but difficult to measure. Dr. Alan Wein analyzes the topic, beginning with the initial definition of $\mathrm{OAB}$ and moving through its updates, and analyzes the potential problems in the application of this definition in clinical practice.

Dr. Victor Nitti reviews the clinical testing of OAB. Despite its completely clinical definition, some tests may help to diagnose the condition. Their indications and techniques are well analyzed by Dr. Nitti.

Treatment of primary $\mathrm{OAB}$ varies slightly in male and female populations. Dr. Linda Cardozo goes through the particularity of diagnosis and treatment in females, while Dr. Christopher Chapple focuses on these aspects in males.

Finally, considerations of two special populations complete this important review: neurogenic bladder overactivity is deeply analyzed by Dr. Clare Fowler, while Dr. Adrian Wagg describes the best approach in elderly patients.

Any physician dealing in practice with $O A B$ should attentively read this practical section, which provides a most accurate update on this difficult condition.

Competing interests: None declared.

This paper has been peer-reviewed.

Correspondence: Dr. Jacques Corcos, Professor of Surgery/Urology, McGill University, Jewish General Hospital, 3755 Côte-Sainte-Catherine, Montreal, QC; jcorcos@uro.jgh.mcgill.ca 